\title{
Designing image trajectories in the presence of uncertain data for robust visual servoing path-planning
}

\author{
Graziano Chesi
}

\begin{abstract}
Path-planning allows one to steer a camera to a desired location while taking into account the presence of constraints such as visibility, workspace, and joint limits. Unfortunately, the planned path can be significantly different from the real path due to the presence of uncertainty on the available data, with the consequence that some constraints may be not fulfilled by the real path even if they are satisfied by the planned path. In this paper we address the problem of performing robust path-planning, i.e. computing a path that satisfies the required constraints not only for the nominal model as in traditional path-planning but rather for a family of admissible models. Specifically, we consider an uncertain model where the point correspondences between the initial and desired views and the camera intrinsic parameters are affected by unknown random uncertainties with known bounds. The difficulty we have to face is that traditional path-planning schemes applied to different models lead to different paths rather than to a common and robust path. To solve this problem we propose a technique based on polynomial optimization where the required constraints are imposed on a number of trajectories corresponding to admissible camera poses and parameterized by a common design variable. The planned image trajectory is then followed by using an IBVS controller. Simulations carried out with all typical uncertainties that characterize a real experiment illustrate the proposed strategy and provide promising results.
\end{abstract}

Index Terms - Visual servoing, Eye-in-hand, Path-planning, Uncertainty, Robustness.

\section{INTRODUCTION}

An important task in robotics consists of automatically positioning a robot by using visual feedback. This task, known as visual servoing, has received a considerable attention due to its wide range of applications. In particular, eye-in-hand visual servoing considers the problem of steering a camera mounted on the end-effector of a robot from an initial to a desired location via a closed-loop control based on the image projections of some object features in the current and desired views. See [1]-[4] for details and classifications. Several methods have been proposed for addressing this task, such as position-based visual servoing (see e.g. [5]), imagebased visual servoing (see e.g. [6]), 2 1/2 D visual servoing [7]. Other methods have proposed partition of the degrees of freedoms (see e.g. [8]), global motion plan via navigation functions (see e.g. [9]), control invariant w.r.t. intrinsic parameters (see e.g. [10]), use of complex image features via image moments (see e.g. [11]), switching strategies for ensuring the visibility constraint (see e.g. [12]), generation of circular-like trajectories for minimizing the trajectory length

G. Chesi is with the Department of Electrical and Electronic Engineering, University of Hong Kong. Contact information: please see http://www.eee.hku.hk/ chesi (see e.g. [13]), and control despite visibility changes (see e.g. [14]).

In order to take into account constraints, for example on the field of view of the camera and on the joints of the robot, path-planning methods have been proposed, see for instance [15]-[19]. These methods typically compute offline a path for reaching the desired location from the initial one based on the available data of the problem, such as the image correspondences among initial and desired views and the camera parameters, and then attempt to control the robot so that it follows such a path. Unfortunately, the planned path can be significantly different from the real path due to the presence of uncertainty on the available data, with the consequence that some constraints may be not fulfilled by the real path. See for instance [20] about the effects of uncertainty.

In this paper we investigate this problem, i.e. performing robust path-planning in the presence of uncertain data. Specifically, we consider that the point correspondences between initial and desired views and the camera intrinsic parameters are affected by unknown random uncertainties with known bounds. The difficulty we have to face is that traditional path-planning schemes applied to different models lead to different paths rather than to a common and robust path. In order to address this problem, each trajectory is parameterized through polynomials by a possible camera pose and by a design variable which is common to all trajectories. We hence estimate the set of admissible camera poses, and impose the required constraints on each trajectory corresponding to each of these camera poses. This allows us for the computation of the sought robust trajectory via the determination of a common design variable that satisfies all constraints on all trajectories so parameterized. The found image trajectory is then followed by using an IBVS controller. The proposed strategy is illustrated through simulations carried out with all typical uncertainties that characterize a real experiment, and the obtained results are satisfactory and promising.

The paper is organized as follows. Section II introduces the problem formulation. Section III presents the proposed path-planning scheme for uncertain data. Section IV presents the simulation results in ideal and in real conditions. Lastly, Section V concludes the paper with some final remarks.

\section{PRoblem DEFINITION}

\section{A. Notation and standard path-planning problem}

The notation exploited in this paper is as follows:

- $S O(3)$ : set of all rotation matrices of size $3 \times 3$; 
- 0: null vector/matrix (size specified by the context);

- 1: vector/matrix with all components equal to 1 (size specified by the context);

- I: identity matrix (size specified by the context);

- $\mathbf{e}_{i}$ : $i$ th column of the identity matrix (size specified by the context);

- $\mathbf{X}^{T}$ : transpose of vector/matrix $\mathbf{X}$;

- $\|\mathbf{X}\|$ : euclidean norm of vector/matrix $\mathbf{X}$;

- $\|\mathbf{X}\|_{\infty}$ : infinity norm of vector/matrix $\mathbf{X}$;

- $[\mathbf{x}]_{\times}$: skew-symmetric matrix of a vector $\mathbf{x} \in \mathbb{R}^{3}$;

- w.r.t., s.t.: with respect to, subject to.

Let $F^{a b s}$ be an absolute frame in the 3D space. We denote with $F=(\mathbf{O}, \mathbf{c})$ the frame of the initial camera of the visual servo system expressed w.r.t. the absolute frame $F^{a b s}$, where $\mathbf{O} \in S O(3)$ is a rotation matrix which defines the orientation, and $\mathbf{c} \in \mathbb{R}^{3}$ is a vector which defines the translation. Similarly we denote with $F^{*}=\left(\mathbf{O}^{*}, \mathbf{c}^{*}\right)$ the frame of the desired camera of the visual servo system.

Let $\mathbf{q}_{1}, \ldots, \mathbf{q}_{n} \in \mathbb{R}^{3}$ be a set of 3D points expressed w.r.t. the absolute frame $F^{a b s}$ and lying in the field of view of the camera in the initial and desired locations. The $i$ th 3D point $\mathbf{q}_{i}$ projects onto the camera frames $F$ and $F^{*}$ at the points $\mathbf{p}_{i}=\left(p_{i, 1}, p_{i, 2}, 1\right)^{T} \in \mathbb{R}^{3}$ and $\mathbf{p}_{i}^{*}=\left(p_{i, 1}^{*}, p_{i, 2}^{*}, 1\right)^{T} \in \mathbb{R}^{3}$, expressed in homogeneous coordinates and given by

$$
\begin{aligned}
d_{i} \mathbf{p}_{i} & =\mathbf{A O}^{T}\left(\mathbf{q}_{i}-\mathbf{c}\right) \\
d_{i}^{*} \mathbf{p}_{i}^{*} & =\mathbf{A O}^{* T}\left(\mathbf{q}_{i}-\mathbf{c}^{*}\right)
\end{aligned}
$$

where $d_{i}, d_{i}^{*}$ are the depths of the point w.r.t. $F$ and $F^{*}$, and $\mathbf{A} \in \mathbb{R}^{3 \times 3}$ is the upper-triangular matrix containing the camera intrinsic parameters:

$$
\mathbf{A}=\left(\begin{array}{ccc}
A_{1} & A_{2} & A_{3} \\
0 & A_{4} & A_{5} \\
0 & 0 & 1
\end{array}\right)
$$

being $A_{1}, A_{4} \in \mathbb{R}$ the focal lengths, $A_{3}, A_{5} \in \mathbb{R}$ the coordinates of the principal point, and $A_{2} \in \mathbb{R}$ the aspect ratio.

Let us gather the available point correspondences between the initial and desired views into the vectors

$$
\begin{aligned}
\mathbf{p} & =\left(p_{1,1}, p_{1,2}, \ldots, p_{n, 1}, p_{n, 2}\right)^{\prime} \\
\mathbf{p}^{*} & =\left(p_{1,1}^{*}, p_{1,2}^{*}, \ldots, p_{n, 1}^{*}, p_{n, 2}^{*}\right)^{\prime} .
\end{aligned}
$$

A standard path-planning problem can be formulated as follows: given an estimate of the vectors $\mathbf{p}, \mathbf{p}^{*}$ and an estimate of the intrinsic parameters matrix $\mathbf{A}$, compute a trajectory along which the camera reaches the desired location while fulfilling a set of constraints such as visibility, workspace, and joint constraints.

\section{B. Path-planning problem for uncertain systems}

In this paper we want to address the path-planning problem in the presence of uncertainties on the available data. Hence, we consider the uncertain model where only an estimate of the image point correspondences $\mathbf{p}, \mathbf{p}^{*}$ is available, that we denote from now on as $\hat{\mathbf{p}}, \hat{\mathbf{p}}^{*}$. Such an estimate is related to the true value $\mathbf{p}, \mathbf{p}^{*}$ by the relationship

$$
\begin{aligned}
\hat{\mathbf{p}} & =\mathbf{p}+\mathbf{n} \\
\hat{\mathbf{p}}^{*} & =\mathbf{p}^{*}+\mathbf{n}^{*}
\end{aligned}
$$

where $\mathbf{n}, \mathbf{n}^{*} \in \mathbb{R}^{2 n}$ are random variables with uniform distribution representing the image noise (for instance due to image quantization, lighting, features extraction, etc...) and which are bounded by

$$
\begin{aligned}
\|\mathbf{n}\|_{\infty} & \leq \eta \\
\left\|\mathbf{n}^{*}\right\|_{\infty} & \leq \eta
\end{aligned}
$$

where $\eta \in \mathbb{R}$ is the image noise intensity. Similarly, we consider that the only an estimate of the intrinsic parameters matrix $\mathbf{A}$ is available, and we denote such an estimate as $\hat{\mathbf{A}}$. The relationship between $\hat{\mathbf{A}}$ and $\mathbf{A}$ is given by

$$
\begin{aligned}
\hat{\mathbf{A}} & =\mathbf{A}+\boldsymbol{\Lambda} \\
\boldsymbol{\Lambda} & =\left(\begin{array}{ccc}
\lambda_{1} & \lambda_{2} & \lambda_{3} \\
0 & \lambda_{4} & \lambda_{5} \\
0 & 0 & 0
\end{array}\right)
\end{aligned}
$$

where $\lambda_{1}, \ldots, \lambda_{5} \in \mathbb{R}$ are random variables with uniform distribution in the intervals

$$
\lambda_{i} \in\left[\lambda_{i}^{-}, \lambda_{i}^{+}\right]
$$

for some limits $\lambda_{1}^{-}, \lambda_{1}^{+}, \ldots, \lambda_{5}^{-}, \lambda_{5}^{+} \in \mathbb{R}$.

The path-planning problem for the uncertain model (5)-(9) can be formulated as follows: given

1) an estimate $\hat{\mathbf{p}}, \hat{\mathbf{p}}^{*}$ of the image point correspondences;

2) an estimate $\hat{\mathbf{A}}$ of the intrinsic parameters matrix;

3) the intensity $\eta$ of the image noise and the limits $\lambda_{1}^{-}, \lambda_{1}^{+}, \ldots, \lambda_{5}^{-}, \lambda_{5}^{+}$of the calibration parameters,

then compute a trajectory along which the camera reaches the desired location while fulfilling a set of constraints such as visibility, workspace, and joint constraints for all possible uncertainties, i.e. for all

$$
\mathbf{n}, \mathbf{n}^{*}, \boldsymbol{\Lambda} \text { fulfilling (6) and (9). }
$$

In the sequel it will be supposed without loss of generality that $F$ coincides with $F^{a b s}$, i.e. $\mathbf{O}=\mathbf{I}$ and $\mathbf{c}=\mathbf{0}$.

\section{ROBUST PATH-PLANNING}

The difficulty we have to face is that any value of the uncertainty $\mathbf{n}, \mathbf{n}^{*}, \boldsymbol{\Lambda}$ determines a different camera pose and hence a different 3D trajectory, with the consequence that also the image trajectories are different, and hence there is no a common trajectory to follow. In order to address this problem, each trajectory is parameterized by a possible camera pose and by a design variable which is common to all trajectories. This allows us for the computation of the sought robust trajectory via a common design variable that satisfies all constraints on all trajectories so parameterized. The details of this strategy are explained in the sequel.

\section{A. Parameterizing the trajectory}

Any $\mathbf{R} \in S O(3)$ can be expressed through Euler parameters (also known as quaternion) as

$$
\mathbf{R}=\|\mathbf{a}\|^{-2} \boldsymbol{\Omega}(\mathbf{a})
$$

where $\mathbf{a}=\left(a_{1}, \ldots, a_{4}\right)^{T} \in \mathbb{R}^{4}$ is a nonzero vector which represents the Euler parameter of $\mathbf{R}$, and $\boldsymbol{\Omega}(\mathbf{a}) \in \mathbb{R}^{3 \times 3}$ is a quadratic function. This parametrization is complete because 
$\boldsymbol{\Omega}(\mathbf{a})$ is a rotation matrix in $S O(3)$ for any unit-norm vector $\mathbf{a} \in \mathbb{R}^{4}$, and because for any rotation matrix $\mathbf{R} \in S O(3)$ there exists a vector $\mathbf{a} \in \mathbb{R}^{4}$ (in particular with unit norm) such that $\mathbf{R}=\boldsymbol{\Omega}(\mathbf{a})$. We indicate such a vector as

$$
\boldsymbol{\xi}(\mathbf{R})=\mathbf{a}
$$

Let us introduce now the parametrization of the camera trajectory. Let $w \in[0,1]$ be the trajectory abscissa, with $w=0$ indicating the initial location and $w=1$ the desired location. Let $(\mathbf{S}, \mathbf{u}) \in S O(3) \times \mathbb{R}^{3}$ be the final camera pose of the trajectory expressed w.r.t. $F$. Clearly such a value should be the camera pose between $F$ and $F^{*}$, which is

$$
\begin{aligned}
& \mathbf{S}=\mathbf{O}^{T} \mathbf{O}^{*} \\
& \mathbf{u}=\frac{\mathbf{O}^{T}\left(\mathbf{c}^{*}-\mathbf{c}\right)}{\left\|\mathbf{O}^{T}\left(\mathbf{c}^{*}-\mathbf{c}\right)\right\|} .
\end{aligned}
$$

Due to the presence of uncertainties, $(\mathbf{S}, \mathbf{u})$ is unknown, and for the moment we consider that $(\mathbf{S}, \mathbf{u})$ is a generic camera pose. We will consider its estimation in Section III-B.

Let us denote with $F(w, \mathbf{S}, \mathbf{u})$ be the camera frame along the trajectory parameterized by the trajectory abscissa $w$ and the final camera pose $(\mathbf{S}, \mathbf{u})$. We denote the camera pose of $F(w, \mathbf{S}, \mathbf{u})$ w.r.t. $F$ as

$$
(\mathbf{R}(w, \mathbf{S}), \mathbf{t}(w, \mathbf{u})) \in S O(3) \times \mathbb{R}^{3} \quad \forall w \in[0,1]
$$

where $\mathbf{R}(w, \mathbf{S}), \mathbf{t}(w, \mathbf{u})$ are functions to be determined. At the extreme points of the trajectory this pose must satisfy

$$
\left\{\begin{array}{l}
(\mathbf{R}(0, \mathbf{S}), \mathbf{t}(0, \mathbf{u}))=(\mathbf{I}, \mathbf{0}) \\
(\mathbf{R}(1, \mathbf{S}), \mathbf{t}(1, \mathbf{u}))=(\mathbf{S}, \mathbf{u})
\end{array}\right.
$$

Now, we want to parameterize the camera pose $(\mathbf{R}(w, \mathbf{S}), \mathbf{t}(w, \mathbf{u}))$ via polynomials. In particular we use polynomials of degree $\delta_{M}$ for the Euler parameters of the rotation and degree $\delta_{N}$ for the translation, according to

$$
\begin{aligned}
\mathbf{a}(w, \mathbf{S}) & =\tilde{\mathbf{M}}\left(w^{\delta_{M}}, w^{\delta_{M}-1}, \ldots, w, 1\right)^{T} \\
\mathbf{t}(w, \mathbf{u}) & =\tilde{\mathbf{N}}\left(w^{\delta_{N}}, w^{\delta_{N}-1}, \ldots, w, 1\right)^{T}
\end{aligned}
$$

where $\tilde{\mathbf{M}} \in \mathbb{R}^{4 \times \delta_{M}+1}$ and $\tilde{\mathbf{N}} \in \mathbb{R}^{3 \times \delta_{N}+1}$. The pose boundary conditions (16) are satisfied if and only if $\tilde{\mathbf{M}}$ and $\tilde{\mathbf{N}}$ have the form

$$
\begin{aligned}
\tilde{\mathbf{M}} & =\left(\boldsymbol{\xi}(\mathbf{S})-\mathbf{M} \mathbf{1}-\mathbf{e}_{4}, \mathbf{M}, \mathbf{e}_{4}\right) \\
\tilde{\mathbf{N}} & =(\mathbf{u}-\mathbf{N} \mathbf{1}, \mathbf{N}, \mathbf{0})
\end{aligned}
$$

where $\mathbf{M} \in \mathbb{R}^{4 \times \delta_{M}-1}$ and $\mathbf{N} \in \mathbb{R}^{3 \times \delta_{N}-1}$ are free matrices. Hence, taking into account (18), we have that (17) becomes

$$
\begin{aligned}
\mathbf{a}(w, \mathbf{S})= & \left(\boldsymbol{\xi}(\mathbf{S})-\mathbf{M} \mathbf{1}-\mathbf{e}_{4}, \mathbf{M}, \mathbf{e}_{4}\right) \\
\mathbf{t}(w, \mathbf{u})= & \left(w^{\delta_{M}}, w^{\delta_{M}-1}, \ldots, w, 1\right)^{T} \\
& \cdot\left(w^{\delta_{N}}, w^{\delta_{N}-1}, \ldots, w, 1\right)^{T} .
\end{aligned}
$$

Therefore, the camera pose of $F(w, \mathbf{S}, \mathbf{u})$ is parameterized by the trajectory abscissa $w$, the final camera pose $(\mathbf{S}, \mathbf{u})$, and the matrices $\mathbf{M}$ and $\mathbf{N}$.

\section{B. Estimating the admissible camera poses}

The next step consists of estimating the admissible camera poses in our uncertain model. Before doing this, let us briefly review how the camera pose is defined and computed for the nominal value of the data $\mathbf{p}, \mathbf{p}^{*}$ and $\mathbf{A}$.

Let $(\mathbf{S}, \mathbf{u})$ be the camera pose between $F$ and $F^{*}$ defined as in (13)-(14). Given $\mathbf{p}, \mathbf{p}^{*}$ and $\mathbf{A}$, the pair $(\mathbf{S}, \mathbf{u})$ can be estimated through the essential matrix algorithm or the homography matrix algorithm relative to a virtual plane in the case of non-coplanar features supposing $n \geq 8$. If the features are known to be coplanar, the camera pose can be computed through the homography matrix algorithm if $n \geq$ 4. See for instance [21]-[24] and references therein. These procedures provide a normalized translational component if no additional information is available but $\mathbf{p}, \mathbf{p}^{*}$ and $\mathbf{A}$ because, in such a case, the translation can be computed only up to a scale factor which stands for the unknown distance between the origins of the $F$ and $F^{*}$. We indicate the estimate of the camera pose returned by any of these algorithms as

$$
\left(E_{r}\left(\mathbf{p}, \mathbf{p}^{*}, \mathbf{A}\right), E_{t}\left(\mathbf{p}, \mathbf{p}^{*}, \mathbf{A}\right)\right) \in S O(3) \times \mathbb{R}^{3} .
$$

Now, let us consider the computation of the admissible camera poses in the uncertain model (5)-(9). This is a difficult problem because one should, first, repeat the camera pose estimation an infinite number of times for all possible true values of the image point correspondences and camera intrinsic parameters, and, second, describe the set of camera poses so found in order to handle it in the path-planning.

In order to cope with these difficulties, we estimate the camera pose only for the extreme values of the uncertainty. The idea behind this choice is that the extreme values of the uncertainty will estimate reasonably well the extreme values of the admissible camera poses, which delimit the whole set of the admissible camera poses and, therefore, can be used for robust path-planning.

Specifically, let $V$ be the set

$$
V=\left\{\left(\mathbf{n}, \mathbf{n}^{*}, \boldsymbol{\Lambda}\right): n_{i}, n_{i}^{*} \in\{-\eta, \eta\}, \lambda_{i} \in\left\{\lambda_{i}^{-}, \lambda_{i}^{+}\right\}\right\}
$$

which is the set of vertices of the hyper-rectangle describing the uncertainty. Let $v_{1}, \ldots, v_{m} \in V$ be a set of triplets contained in $V$ for some integer $m$, and let us denote the $i$ th triplet $v_{i}$ as $v_{i}=\left(\mathbf{n}_{i}, \mathbf{n}_{i}^{*}, \boldsymbol{\Lambda}_{i}\right)$. Then, we define the camera pose $\left(\mathbf{S}_{i}, \mathbf{u}_{i}\right) \in S O(3) \times \mathbb{R}^{3}$ associated with the triplet $v_{i}$ as

$$
\begin{aligned}
& \mathbf{S}_{i}=E_{r}\left(\hat{\mathbf{p}}-\mathbf{n}, \hat{\mathbf{p}}^{*}-\mathbf{n}^{*}, \hat{\mathbf{A}}-\mathbf{\Lambda}\right) \\
& \mathbf{u}_{i}=E_{t}\left(\hat{\mathbf{p}}-\mathbf{n}, \hat{\mathbf{p}}^{*}-\mathbf{n}^{*}, \hat{\mathbf{A}}-\mathbf{\Lambda}\right) .
\end{aligned}
$$

The camera poses $\left(\mathbf{S}_{1}, \mathbf{u}_{1}\right), \ldots,\left(\mathbf{S}_{m}, \mathbf{u}_{m}\right)$ have the role to bound the set of admissible camera poses in the uncertain model (5)-(9), and are obtained by computing the camera pose associated with each triplet $v_{1}, \ldots, v_{m}$.

The triplets $v_{1}, \ldots, v_{m}$ are randomly picked up from $V$ with the constraint that they must be distinct (in order to avoid useless copies). The number $m$ of these triplets represents hence a trade-off between accuracy of the estimation 
of the set of admissible camera poses and complexity of the procedure, and can be arbitrarily selected.

\section{Determining the image trajectory}

In this section we describe how typical constraints are imposed on the parameterized trajectory for our uncertain model.

Let us consider first the visibility constraint. The image projection of the $j$ th object point onto the camera frame $F\left(w, \mathbf{S}_{i}, \mathbf{u}_{i}\right)$ of the $i$ th camera pose can be expressed in terms of $\mathbf{a}\left(w, \mathbf{S}_{i}\right)$ and $\mathbf{t}\left(w, \mathbf{u}_{i}\right)$ as

$$
\begin{gathered}
d_{i, j}\left(w, \mathbf{S}_{i}, \mathbf{u}_{i}\right) \mathbf{p}_{i, j}\left(w, \mathbf{S}_{i}, \mathbf{u}_{i}\right)= \\
\mathbf{A} \boldsymbol{\Omega}\left(\mathbf{a}\left(w, \mathbf{S}_{i}\right)\right)^{T}\left(\mathbf{v}_{i, j}\left(w, \mathbf{S}_{i}, \mathbf{u}_{i}\right)-\mathbf{t}\left(w, \mathbf{u}_{i}\right)\right)
\end{gathered}
$$

where $\mathbf{p}_{i, j}\left(w, \mathbf{S}_{i}, \mathbf{u}_{i}\right) \in \mathbb{R}^{3}$ is the image point, $d_{i, j}(w, \mathbf{S}, \mathbf{u}) \in \mathbb{R}$ is the point depth, and $\mathbf{v}_{i, j}\left(w, \mathbf{S}_{i}, \mathbf{u}_{i}\right) \in$ $\mathbb{R}^{3}$ is a parameter-dependent reconstruction of the $j$ th $3 \mathrm{D}$ point $\mathbf{q}_{j}$ associated with the camera pose $\left(\mathbf{S}_{i}, \mathbf{u}_{i}\right)$ which ensures the fulfillment of the boundary conditions

$$
\left\{\begin{array}{l}
\mathbf{p}_{i, j}\left(0, \mathbf{S}_{i}, \mathbf{u}_{i}\right)=\hat{\mathbf{p}}_{i}-\mathbf{n}_{i} \\
\mathbf{p}_{i, j}\left(1, \mathbf{S}_{i}, \mathbf{u}_{i}\right)=\hat{\mathbf{p}}_{i}^{*}-\mathbf{n}_{i}^{*}
\end{array}\right.
$$

where $\mathbf{n}_{i}, \mathbf{n}_{i}^{*}$ are the vectors of image noise who have determined $\left(\mathbf{S}_{i}, \mathbf{u}_{i}\right)$ in (22)-(23). Such a reconstruction $\mathbf{v}_{i, j}\left(w, \mathbf{S}_{i}, \mathbf{u}_{i}\right)$ has the form

$$
\mathbf{v}_{i, j}\left(w, \mathbf{S}_{i}, \mathbf{u}_{i}\right)=\mathbf{v}_{i, j, 0}+\mathbf{v}_{i, j, 1} w
$$

where $\mathbf{v}_{i, j, 0}, \mathbf{v}_{i, j, 1} \in \mathbb{R}^{3}$ are suitable vectors which can be found in closed formula via standard least-squares for given $\mathbf{S}_{i}, \mathbf{u}_{i}, \hat{\mathbf{p}}_{i}-\mathbf{n}_{i}, \hat{\mathbf{p}}_{i}^{*}-\mathbf{n}_{i}^{*}, \hat{\mathbf{A}}-\boldsymbol{\Lambda}$. Hence, $\mathbf{p}_{i, j}\left(w, \mathbf{S}_{i}, \mathbf{u}_{i}\right)$ is

$$
\mathbf{p}_{i, j}\left(w, \mathbf{S}_{i}, \mathbf{u}_{i}\right)=\frac{1}{b_{i, j, 3}\left(w, \mathbf{S}_{i}, \mathbf{u}_{i}\right)}\left(\begin{array}{c}
b_{i, j, 1}\left(w, \mathbf{S}_{i}, \mathbf{u}_{i}\right) \\
b_{i, j, 2}\left(w, \mathbf{S}_{i}, \mathbf{u}_{i}\right) \\
b_{i, j, 3}\left(w, \mathbf{S}_{i}, \mathbf{u}_{i}\right)
\end{array}\right)
$$

where

$$
\begin{gathered}
b_{i, j, k}\left(w, \mathbf{S}_{i}, \mathbf{u}_{i}\right)= \\
\mathbf{e}_{k}^{T} \mathbf{A} \boldsymbol{\Omega}\left(\mathbf{a}\left(w, \mathbf{S}_{i}\right)\right)^{T}\left(\mathbf{v}_{i, j}\left(w, \mathbf{S}_{i}, \mathbf{u}_{i}\right)-\mathbf{t}\left(w, \mathbf{u}_{i}\right)\right) .
\end{gathered}
$$

This point lies in the field of view of the camera if and only if there exists $\gamma \geq 0$ such that

$$
\left\{\begin{array}{l}
x_{-} b_{i, j, 3}\left(w, \mathbf{S}_{i}, \mathbf{u}_{i}\right)-b_{i, j, 1}\left(w, \mathbf{S}_{i}, \mathbf{u}_{i}\right) \leq-\gamma \\
x_{+} b_{i, j, 3}\left(w, \mathbf{S}_{i}, \mathbf{u}_{i}\right)-b_{i, j, 1}\left(w, \mathbf{S}_{i}, \mathbf{u}_{i}\right) \geq \gamma \\
y_{-} b_{i, j, 3}\left(w, \mathbf{S}_{i}, \mathbf{u}_{i}\right)-b_{i, j, 2}\left(w, \mathbf{S}_{i}, \mathbf{u}_{i}\right) \leq-\gamma \\
y_{+} b_{i, j, 3}\left(w, \mathbf{S}_{i}, \mathbf{u}_{i}\right)-b_{i, j, 2}\left(w, \mathbf{S}_{i}, \mathbf{u}_{i}\right) \geq \gamma
\end{array}\right.
$$

where $x_{-}, x_{+}, y_{-}, y_{+} \in \mathbb{R}$ are the limits of the image screen, and $\gamma \in \mathbb{R}$ quantifies the feasibility of the constraint.

Let us consider now the workspace constraint. This constraint imposes that the camera must always remain in an allowed region. Let us describe such a region as

$$
G=\left\{\mathbf{x} \in \mathbb{R}^{3}: g_{j}(\mathbf{x}) \geq 0, j=1, \ldots, n_{G}\right\}
$$

where $g_{1}, g_{2}, \ldots: \mathbb{R}^{3} \rightarrow \mathbb{R}$ are polynomials which describe the region w.r.t. the desired camera frame $F^{*}$ (since this is typically a reference location). Then, the camera center of $F\left(w, \mathbf{S}_{i}, \mathbf{u}_{i}\right)$ w.r.t. $F^{*}$ is given by

$$
\boldsymbol{\Omega}\left(\mathbf{a}\left(w, \mathbf{S}_{i}\right)\right)^{T}\left(\mathbf{t}\left(w, \mathbf{u}_{i}\right)-\mathbf{u}_{i}\right)
$$

and, hence, one has to impose that

$$
g_{j}\left(\boldsymbol{\Omega}\left(\mathbf{a}\left(w, \mathbf{S}_{i}\right)\right)^{T}\left(\mathbf{t}\left(w, \mathbf{u}_{i}\right)-\mathbf{u}_{i}\right)\right) \geq 0 .
$$

The robust path-planning problem is hence: maximize $\gamma$ w.r.t. $\mathbf{M}, \mathbf{N}$ such that, $\forall i=1, \ldots, m \forall w \in[0,1]$,

(29) holds $\forall j=1,2$ and (32) holds $\forall j=1, \ldots, n_{G}$

Let us observe that, while each admissible camera pose $\left(\mathbf{S}_{i}, \mathbf{u}_{i}\right)$ leads to a different constraint, the parameters to determine in the path-planning problem (i.e., the matrices $\mathbf{M}, \mathbf{N}$ ) are common to all these constraints. In fact, these matrices describe the shape of each admissible trajectory, which is hence the same for all admissible trajectories. Therefore, the robust path-planning problem (33) defines a trajectory which satisfies the visibility constraint for all considered uncertainties.

Problem (33) is solved by maximizing $\gamma$ w.r.t. $\mathbf{M}, \mathbf{N}$ subject to the constraint (29). Clearly one can terminate the maximization whenever a pair $\mathbf{M}, \mathbf{N}$ guaranteeing (29) with any $\gamma \geq 0$ is found. This problem can be solved through a search where at each iteration one evaluates the roots of one-variable polynomials.

Also, let us observe that, by considering large uncertainties in the model (5)-(7), it is possible that all the admissible constraints cannot be ensured. Nevertheless, the solution returned by (33) provides the trajectory which fulfills (29) with the largest $\gamma$, i.e. the trajectory that better than any other one satisfies all the admissible constraints.

Similarly to what done for the visibility and workspace constraints, one can take into account joint constraints in the robust path-planning problem (33). This part is omitted for conciseness, and the reader is referred to [19] where the construction of such polynomials is described for the standard path-planning case.

Once the the robust path-planning problem (33) has been solved, one builds the image trajectories determined by the found $\mathbf{M}, \mathbf{N}$ and by the camera pose relative to the nominal model, i.e. the image trajectories $\mathbf{p}_{0, j}\left(w, \mathbf{S}_{0}, \mathbf{u}_{0}\right)$ defined in (27) where $\left(\mathbf{S}_{0}, \mathbf{u}_{0}\right)$ is the camera pose corresponding to the available data given by $\mathbf{S}_{0}=E_{r}\left(\hat{\mathbf{p}}, \hat{\mathbf{p}}^{*}, \hat{\mathbf{A}}\right)$ and $\mathbf{u}_{0}=$ $E_{t}\left(\hat{\mathbf{p}}, \hat{\mathbf{p}}^{*}, \hat{\mathbf{A}}\right)$. These image trajectories are lastly tracked by using an IBVS controller.

\section{EXAMPLE}

In this section we illustrate the application of the proposed approach and report some results. Due to lack of time, we cannot present experimental results yet. To cope with this deficiency, we report simulation results obtained while attempting to emulate real situations, i.e. by supposing that all the considered data and the final IBVS controller used to track the planned trajectory are affected by uncertainty.

Let us consider the configuration shown in Figure 1a where the camera in the initial and desired locations is observing the 9 large dots of three dices. The problem is to reach the desired location by avoiding collisions with the 


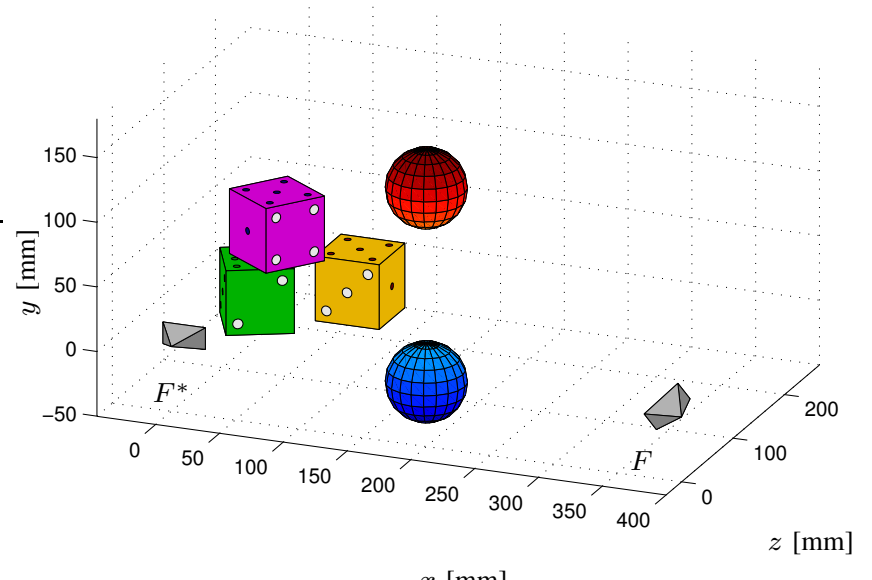

$x[\mathrm{~mm}]$

(a)

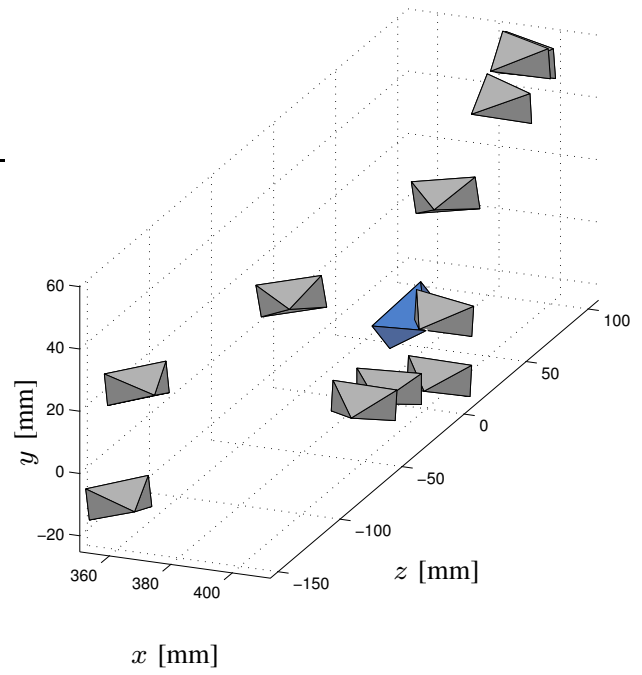

(b)

Fig. 1. (a) Camera frames $F, F^{*}$, observed objects, and obstacles. (b) Some admissible camera poses in the uncertainty model: each camera is an admissible location of the initial frame w.r.t. $F^{*}$ (the true initial location is the blue camera).

two spheres, which represent two obstacles, and by avoiding occlusions due to these two spheres.

In order to emulate as much as possible real situations, we consider the following uncertainties:

1) (Image noise) The available points $\hat{\mathbf{p}}, \hat{\mathbf{p}}^{*}$ are obtained by adding random variables with uniform distribution in the interval $[-1,1]$ pixels to each component of the true values $\mathbf{p}, \mathbf{p}^{*}$. The screen size is $640 \times 480$ pixels.

2) (Intrinsic calibration errors) The available estimate of the intrinsic parameters matrix and its true value are

$$
\hat{\mathbf{A}}=\left(\begin{array}{ccc}
843 & 0 & 335 \\
0 & 760 & 226 \\
0 & 0 & 1
\end{array}\right), \mathbf{A}=\left(\begin{array}{ccc}
800 & 0 & 320 \\
0 & 800 & 240 \\
0 & 0 & 1
\end{array}\right) \text {. }
$$

3) (Uncertain point depth) The available point depth in the current camera frame needed to define the Jacobian in the IBVS controller is obtained via an object reconstruction through the current camera pose provided by the essential matrix, and it is hence affected by the supposed image noise and intrinsic calibration errors;

4) (Extrinsic calibration errors) The available estimate of the extrinsic camera parameters used to apply IBVS is

$$
\boldsymbol{\theta}_{E}=(-5,5,5)^{T} \mathrm{deg}, \quad \mathbf{t}_{E}=(10,-10,10)^{T} \mathrm{~mm}
$$

where $\boldsymbol{\theta}_{E}, \mathbf{t}_{E}$ are rotation (in exponential coordinates) and translation of the camera w.r.t. the end-effector directly controlled (true values: $\boldsymbol{\theta}_{E}=\mathbf{0}, \mathbf{t}_{E}=\mathbf{0}$ ).

Now, in order to plan a robust camera trajectory we introduce the uncertain model (5)-(7), for example with

$$
\eta=1 \text { pixel }, \quad \lambda_{i}^{-}=90 \% \hat{A}_{i}, \quad \lambda_{i}^{+}=110 \% \hat{A}_{i}
$$

hence considering uncertainty up to \pm 1 pixel on each component of each image point, and up to $\pm 10 \%$ on each camera intrinsic parameter.

The next step is to estimate the admissible camera poses. We select $m=20$ and compute the camera poses $\left(\mathbf{S}_{i}, \mathbf{u}_{i}\right)$ in (22)-(23) by using the essential matrix algorithm without any CAD model of the object. Figure $1 \mathrm{~b}$ shows the admissible locations of the initial frame w.r.t. $F^{*}$ corresponding to some of these camera poses.

Hence, we build the visibility and workspace constraints in (29) and (32). In particular, we model the two spheres as two redundant boxes in order to avoid that the camera may pass behind them and hence lose the object points due to occlusions. In order to do this, we assume that the distance between two object points is known in order to estimate the norm of the translation.

Then, we solve the robust path-planning in (33) for $\delta_{M}=$ $\delta_{N}=2$, hence finding the matrices $\mathbf{M}, \mathbf{N}$ which determine the trajectory (the computational time is less than 4 seconds on a standard PC). We track this trajectory by using the IBVS controller in [15] implemented with wrong estimates as described above, and we obtain the results shown in Figure 2 which reports the followed 3D trajectory, camera view, and camera coordinates along the trajectory. As we can see, the imposed constraints are satisfactorily fulfilled in spite of all present uncertainties, in particular the camera reaches the desired location by satisfying visibility and avoiding collisions with/occlusions from the two spheres.

It is worthwhile to mention that, by performing the pathplanning by using only the nominal model, we find that the followed $3 \mathrm{D}$ trajectory collides with one of the spheres.

\section{CONCLUSiON}

We have addressed the problem of performing robust pathplanning, i.e. determining a trajectory that satisfies typical constraints in the presence of bounded random uncertainty on the point correspondences and camera intrinsic parameters. We have hence proposed a technique for computing a trajectory that satisfies such constraints for a family of admissible models. This technique is based firstly on the estimation of the set of admissible camera poses, and secondly on the imposition of the required constraints on different trajectories corresponding to different admissible camera poses and parameterized by a common design variable. We 


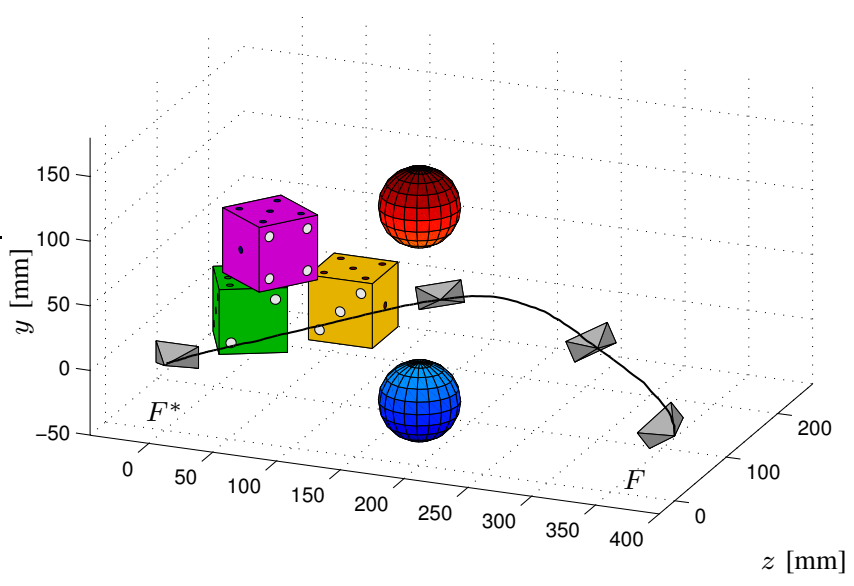

$x[\mathrm{~mm}]$

(a)

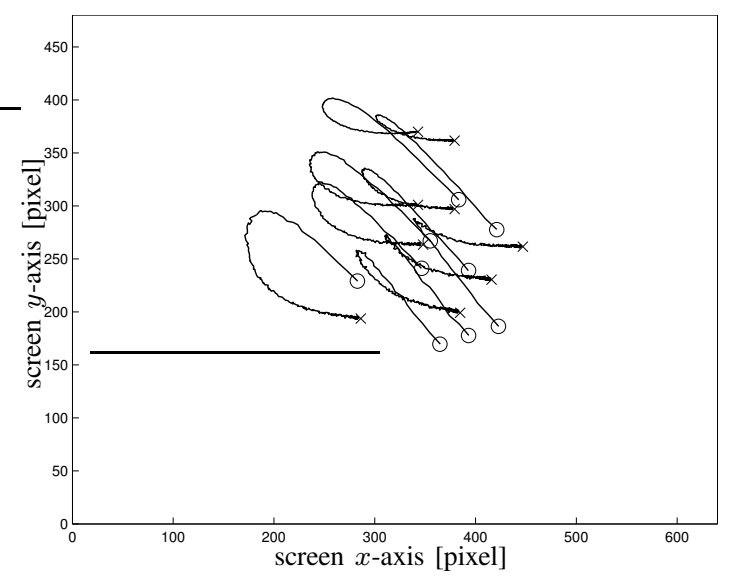

(b)

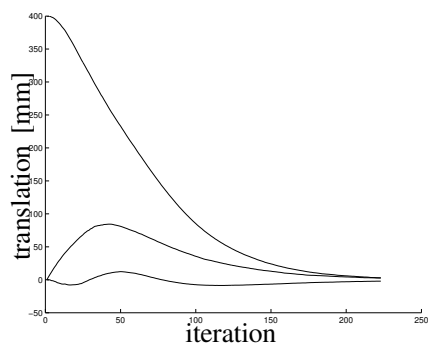

(c)

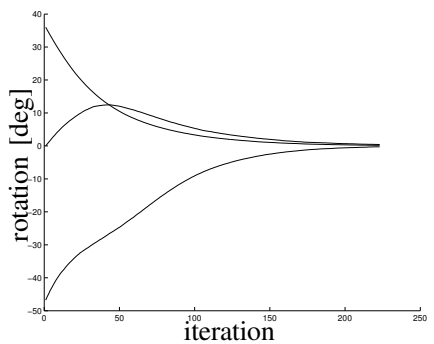

(d)
Fig. 2. (a) Followed 3D trajectory. (b) Camera view. (c-d) Camera coordinates expressed in $F^{*}$.

have illustrated the proposed technique through simulations carried out with all typical uncertainties that characterize a real experiment, which have provided satisfactory and promising results.

We believe that the ideas introduced in this paper to provide a robust trajectory may find useful applications also in other visual servoing problems where the available data are always affected by uncertainty.

\section{ACKNOWLEDGEMENT}

The authors would like to thank the Associate Editor and Reviewers for their time and comments.

\section{REFERENCES}

[1] S. Hutchinson, G. Hager, and P. Corke, "A tutorial on visual servo control," IEEE Trans. on Robotics and Automation, vol. 12, no. 5, pp. 651-670, 1996.

[2] K. Hashimoto, "A review on vision-based control of robot manipulators," Advanced Robotics, vol. 17, no. 10, pp. 969-991, 2003.

[3] F. Chaumette and S. Hutchinson, "Visual servo control, part I: Basic approaches," IEEE Robotics and Automation Magazine, vol. 13, no. 4, pp. 82-90, 2006.

[4] — "Visual servo control, part II: Advanced approaches," IEEE Robotics and Automation Magazine, vol. 14, no. 1, pp. 109-118, 2007.

[5] B. Thuilot, P. Martinet, L. Cordesses, and J. Gallice, "Position based visual servoing: keeping the object in the field of vision," in Proc. IEEE Int. Conf. on Robotics and Automation, Washington, D.C., 2002, pp. 1624-1629.

[6] F. Chaumette, "Potential problems of stability and convergence in image-based and position-based visual servoing," in The confluence of vision and control, G. H. D. Kriegman and A. Morse, Eds. SpringerVerlag, 1998, pp. 66-78.

[7] E. Malis, F. Chaumette, and S. Boudet, "2 1/2 D visual servoing," IEEE Trans. on Robotics and Automation, vol. 15, no. 2, pp. 238250, 1999.

[8] P. I. Corke and S. Hutchinson, "A new partitioned approach to imagebased visual servo control," IEEE Trans. on Robotics and Automation, vol. 17 , no. 4, pp. 507-515, 2001.

[9] N. J. Cowan and D. E. Chang, "Geometric visual servoing," IEEE Trans. on Robotics, vol. 21, no. 6, pp. 1128-1138, 2005.

[10] E. Malis, "Visual servoing invariant to changes in camera-intrinsic parameters," IEEE Trans. on Robotics and Automation, vol. 20, no. 1, pp. 72-81, 2004.

[11] O. Tahri and F. Chaumette, "Point-based and region-based image moments for visual servoing of planar objects," IEEE Trans. on Robotics, vol. 21, no. 6, pp. 1116-1127, 2005.

[12] G. Chesi, K. Hashimoto, D. Prattichizzo, and A. Vicino, "Keeping features in the field of view in eye-in-hand visual servoing: a switching approach," IEEE Trans. on Robotics, vol. 20, no. 5, pp. 908-913, 2004.

[13] G. Chesi and A. Vicino, "Visual servoing for large camera displacements," IEEE Trans. on Robotics, vol. 20, no. 4, pp. 724-735, 2004.

[14] N. Garcia-Aracil, E. Malis, R. Aracil-Santonja, and C. Perez-Vidal, "Continuous visual servoing despite the changes of visibility in image features," IEEE Trans. on Robotics, vol. 21, no. 6, pp. 1214-1220, 2005.

[15] Y. Mezouar and F. Chaumette, "Path planning for robust image-based control," IEEE Trans. on Robotics and Automation, vol. 18, no. 4, pp. 534-549, 2002.

[16] J. Park and M. Chung, "Path planning with uncalibrated stereo rig for image-based visual servoing under large pose discrepancy," IEEE Trans. on Robotics and Automation, vol. 19, no. 2, pp. 250-258, 2003.

[17] L. Deng, F. Janabi-Sharifi, and W. J. Wilson, "Hybrid motion control and planning strategy for visual servoing," IEEE Trans. on Industrial Electronics, vol. 52, no. 4, pp. 1024-1040, 2005.

[18] B. Allotta and D. Fioravanti, "3D motion planning for image-based visual servoing tasks," in Proc. IEEE Int. Conf. on Robotics and Automation, Barcelona, Spain, 2005.

[19] G. Chesi and Y. S. Hung, "Global path-planning for constrained and optimal visual servoing," IEEE Trans. on Robotics, vol. 23, no. 5, pp. 1050-1060, 2007.

[20] V. Kyrki, D. Kragic, and H. Christensen, "Measurement errors in visual servoing," Robotics and Autonomous Systems, vol. 54, no. 10, pp. 815$827,2006$.

[21] R. Hartley and A. Zisserman, Multiple view in computer vision. Cambridge University Press, 2000.

[22] E. Malis and F. Chaumette, " 2 1/2 D visual servoing with respect to unknown objects through a new estimation scheme of camera displacement," Int. Journal of Computer Vision, vol. 37, no. 1, pp. 79-97, 2000.

[23] G. Chesi and K. Hashimoto, "A simple technique for improving camera displacement estimation in eye-in-hand visual servoing," IEEE Trans. on Pattern Analysis and Machine Intelligence, vol. 26, no. 9, pp. 1239-1242, 2004

[24] G. Chesi, "Camera displacement via constrained minimization of the algebraic error," IEEE Trans. on Pattern Analysis and Machine Intelligence, vol. 31, no. 2, pp. 370-375, 2009. 